\title{
Aberrant ERG expression cooperates with loss of PTEN to promote cancer progression in the prostate
}

\author{
Brett S Carver ${ }^{1,3}$, Jennifer Tran ${ }^{1}$, Anuradha Gopalan ${ }^{2}$, Zhenbang Chen ${ }^{1,5}$, Safa Shaikh ${ }^{3}$, \\ Arkaitz Carracedo $^{1,5}$, Andrea Alimonti ${ }^{1,5}$, Caterina Nardella ${ }^{1,5}$, Shohreh Varmeh ${ }^{1,5}$, Peter \\ T Scardino $^{3}$, Carlos Cordon-Cardo ${ }^{4}$, William Gerald ${ }^{2}$, and Pier Paolo Pandolfi ${ }^{1,2,5,{ }^{*}}$ \\ ${ }^{1}$ Cancer Biology and Genetics Program, Sloan-Kettering Institute, New York, New York 10021. \\ 2 Department of Pathology, Memorial Sloan-Kettering Cancer Center, New York, New York \\ 10021. \\ ${ }^{3}$ Department of Surgery, Division of Urology, Memorial Sloan-Kettering Cancer Center, New \\ York, New York 10021. \\ 4 Department of Pathology, Columbia University, New York, NY 10032. \\ ${ }^{5}$ Cancer Genetics Program, Beth Israel Deaconess Cancer Center, Department of Medicine and \\ Pathology, Harvard Medical School, Boston MA 02215.
}

\begin{abstract}
Chromosomal translocations involving the $E R G$ locus are frequent events observed in human prostate cancer pathogenesis, however the biologic role of ERG aberrant expression is controversial. ${ }^{1}$ Here we demonstrate that the aberrant expression of ERG is a progression event in prostate tumorigenesis. We find that prostate cancer specimens containing the TMPRSS2:ERG genetic rearrangement are significantly enriched for loss of the tumor suppressor PTEN. In concordance with these findings, over-expression of ERG in the transgenic mouse prostate promotes a marked acceleration and progression of HGPIN to prostatic adenocarcinoma in a Pten heterozygous background. In vitro over-expression of ERG promotes cell migration, a property necessary for tumorigenesis, without affecting proliferation. ADAMTS1 and CXCR4, two candidate genes strongly associated with cell migration are found up-regulated in the presence of ERG over-expression. Thus, ERG plays a distinct role in prostate cancer progression and cooperates with PTEN haploinsufficiency to promote progression of HGPIN to invasive adenocarcinoma.
\end{abstract}

The first recurrent translocation event in prostate cancer was recently described in a seminal study. ${ }^{1}$ It results in the translocation of an ETS transcription factor (ERG or ETVI) to the TMPRSS2 promoter region, which contains androgen responsive elements. ${ }^{1}$ The

TMPRSS:ERG genetic rearrangement is the most common and has been reported to occur in approximately $40 \%$ of primary prostate tumors and results in an aberrant androgen regulated expression of ERG. ${ }^{1-3}$ Additionally, ETS family members ETV1, ETV4, and ETV5 have

\footnotetext{
* To whom correspondence should be addressed: ppandolf@bidmc.harvard.edu .

Author Contributions Brett S Carver designed the study, conducted and supervised all experiments, and wrote the manuscript along with Pier Paolo Pandolfi. Jennifer Tran conducted in vivo and in vitro experiments. Anuradha Gopalan designed and performed the human tissue analyses. Zhenbang Chen provided the prostate tissue from our Pten/p53 prostate conditional null series of mice. Safa Shaikh performed the BPH-1 cell experiments. Arkiatz Carracedo, Andrea Alimonti, Caterina Nardella, Shoreh Varmeh, and Peter T Scardino provided critical discussion on the generation of our mouse model and experimental design. Carlos Cordon-Cardo reviewed our mouse prostate histology. William Gerald supervised Anuradha Gopalan on the human tissue studies and reviewed our mouse prostate histology. Pier Paolo Pandolfi supervised and mentored all work. All authors approved of the final manuscript.
} 
been demonstrated to be genetically rearranged to either androgen regulated or ubiquitous promoter regions resulting in their aberrant expression in prostate cancer. ${ }^{4,5}$

Recently, it has been reported that prostate specific over-expression of ETV1 or ERG results in a very subtle phenotype of hyperplasia and prostatic intra-epithelial neoplasia (PIN), a pre-malignant in situ lesion. ${ }^{6,7}$ These data have been interpreted as an indication of the critical proto-oncogenic role of ERG in tumor initiation. Given the subtle phenotypes observed in the previously published mouse models, and cellular and molecular studies suggesting that ETS over-expression promotes invasive properties without an effect on proliferation, we hypothesized that ERG associated translocations and the resulting aberrant over-expression of the transcription factor may in fact not represent initiation events but rather critical cooperative progression events in prostate tumorigenesis. We here present data in support of this hypothesis that in turn, change our view on the role of ERG aberrant expression in prostate tumorigenesis.

We sought to determine whether $E R G$ genetic rearrangements would be identified in early prostate cancer lesions. We find in fact, that genetic rearrangement of $E R G$ is infrequently found in HGPIN and only in a minority of patients, all of whom also have the translocation present in associated adenocarcinoma of the prostate (Figure 1A). Forty prostate cancer specimens on a tumor tissue microarray were evaluated for the presence of HGPIN with adjacent carcinoma, of which $10 \%$ had evidence of $E R G$ genetic rearrangements as indicated by $E R G$ FISH analysis. All of these cases demonstrated the $E R G$ genetic rearrangement in the adjacent invasive prostate cancer as well, however, the majority of prostate cancer specimens with $E R G$ genetic rearrangements do not display this rearrangement in the associated HGPIN and there were no cases of $E R G$ genetic rearrangements in HGPIN associated with $E R G$ negative cancers. Similarly, Mosquera et al reported that $E R G$ genetic rearrangements were found in only $16 \%$ of all HGPIN lesions and in all cases, the adjacent prostate cancer shared the same $E R G$ fusion pattern. ${ }^{8}$ Furthermore, of the ERG fusion positive cancers, $60 \%$ did not demonstrate ERG genetic rearrangement in the associated HGPIN. ${ }^{8}$ In contrast loss of PTEN has been demonstrated to occur in approximately $45 \%$ of HGPIN. ${ }^{9}$ Therefore, the TMPRSS2:ERG translocation appears to be an early event in human prostate tumorigenesis, but one potentially associated with progression from HGPIN to cancer.

Primary prostate cancers often show genetic loss or alteration (mutation) of at least one PTEN allele in approximately $30 \%$ to $70 \%$ of cases. ${ }^{10,11}$ Our lab has previously established the critical role of Pten loss in prostate tumorigenesis. ${ }^{12-14}$ To evaluate whether loss of PTEN and $E R G$ genetic rearrangements are concomitant events in prostate cancer, specimens were analyzed for $E R G$ genetic rearrangements and PTEN protein expression in consecutive sections from a tumor tissue microarray (Figure 1B). A total of 40 prostate cancer specimens were analyzed and homogenous PTEN protein expression was reduced or absent in 27 (68\%) samples. Homogenous $E R G$ genetic rearrangements as demonstrated by FISH analysis were present in 15 (38\%) samples. Strikingly 14 of the 15 ERG FISH positive samples had reduced or absent PTEN expression. Additionally, King et al find that genomic loss of PTEN and ERG genetic rearrangements are significantly associated genetic events. ${ }^{15}$ Collectively, these and previously published findings, ${ }^{15,16}$ highlight that in human prostate cancer, PTEN loss and ERG genetic rearrangements are concomitant events.

Based on our human data, we explored whether ERG aberrant expression was observed in prostate tumors from our mouse models of prostate cancer. We have previously shown that the prostate conditional knock-out of Pten results in an indolent form of prostate cancer and that concomitant loss of $p 53$ dramatically results in an advanced lethal form of the disease. $13,14 \mathrm{We}$ surprisingly found that the expression of murine Erg is markedly increased at the 
mRNA level in tumors from prostate conditional $\mathrm{Pten}^{-/-} \mathrm{p} 53^{-/-}$mice compared to prostate tumors from $\mathrm{Pten}^{-/-} \mathrm{p} 53^{+/+}$mice and control prostates (Figure 2A). The upregulation of $\mathrm{Erg}$, appears to be selected for during tumor evolution, as Erg transcript levels were similar between primary MEF cells of the various genotypes (data not shown).

Given that genetic rearrangements involving ERG and significantly associated with PTEN loss in human prostate cancer, we sought to determine whether aberrant prostatic expression of $E R G$ would cooperate with Pten loss to promote prostate cancer development and progression. Pten heterozygous mice develop high grade PIN with an increased proliferative rate after a long latency with incomplete penetrance. ${ }^{12}$ Mice expressing $E R G$ under the control of the probasin (ARR2PB) promoter were generated (Supplementary Figure 1). While the PB-ERG mice did not develop a significant phenotype throughout their lives, we found that prostate specific expression of ERG cooperates with Pten haploinsufficiency, resulting in a highly penetrant phenotype of invasive prostatic adenocarcinoma and a reduction in cancer latency (Figure 2B). By 6 months of age, Pten $^{+/-}$PB-ERG mice displayed multifocal prostatic adenocarcinoma with complete penetrance (Figure 2B). Conversely, control Pten ${ }^{+-}$mice do not develop prostatic adenocarcinoma at any age and display high-grade PIN starting at approximately 8 months of age. ${ }^{12}$ Furthermore, and strikingly, $\mathrm{Pten}^{+/-}$PB-ERG mice demonstrated a rapid acceleration of disease with overt HGPIN starting at 2 months of age and progressing to invasive carcinoma by 6 months of age (Figure 2B). In the pre-neoplastic prostate glands of these mice, ERG over-expression was not associated with a proliferative advantage, while $\mathrm{Pten}^{+/-}$mice displayed a significant increase in cell proliferation which was not different in the Pten $^{+/-}$PB-ERG compound mice (Figure 2C).

We next assessed whether prostate specific over-expression of ERG would affect Pten levels or function. The levels of Pten and Akt/pAkt were similar between the two genotypes by immunohistochemical staining of histologically normal prostate glands and qRT-PCR analysis (Figure 2D and not shown). Therefore, prostate specific over-expression of ERG does not appear to directly affect Pten levels, thus likely promoting prostate tumorigenesis through a complementary pathway.

To explore the mechanisms by which ERG may be promoting the development and progression of prostate tumorigenesis, we next studied in vitro ERG-dependent cellular processes. To this end, stable cell lines over-expressing ERG, a constitutively active AKT (AKT-1), or vector control were generated from BPH-1 cells. Western blot analysis confirmed ERG (flag) expression, constitutive expression and activation of AKT, and phosphorylation status of S6 in these cell lines (Figure 3A). In agreement with what was observed in vivo in the prostate of transgenic mice, we found no significant difference in cellular proliferation when ERG was over-expressed (Figure 3B). By contrast, overexpression of constitutively active AKT resulted in a proliferative advantage, which was not augmented by ERG over-expression (Figure 3B). However, we did observe a striking increase in cell migration when ERG was over-expressed compared to vector control, which was not enhanced by constitutive AKT activation (Figure $3 \mathrm{C}$ ). We subsequently confirmed that ERG over-expression did not alter cell proliferation, but promoted cell migration in both primary MEF and PC3 cells (data not shown).

To determine the basis underlying the role of ERG in the regulation of cell migration, a microarray expression analysis was performed comparing 293 HEK cells over-expressing ERG or vector control. Two independent experiments were conducted and analyzed for genes with a 2 fold or greater difference across the samples (Table 1). A total of 167 differentially expressed genes were identified (Supplementary Figure 2A). Two genes, CXCR4 and ADAMTS1, which are implicated in promoting cell migration and invasion were 
found to be transcriptionally up-regulated upon ERG expression. ${ }^{17,18} \mathrm{CXCR} 4$ is a chemokine receptor implicated in cell migration, cell adhesion, angiogenesis, and the homing of hemopoietic cells. Additionally, CXCR4 has been shown to be over-expressed in some carcinomas such as breast, kidney, and prostate cancer and transcriptionally regulated by Ets-1 under conditions of hypoxia. ${ }^{19-23}$ These two putative candidates were further validated by quantitative RT-PCR and confirmed to be up-regulated in cells over-expressing ERG (Supplementary Figure 2B).

To determine whether these genes are direct ERG targets we searched the $5^{\prime}$ upstream promoter region of $C X C R 4$ and ADAMTS1 for putative ETS binding sites. For CXCR4 when analyzing the upstream $-3 \mathrm{~kb}$ promoter region two ETS binding sites were identified within $113 \mathrm{bp}$ of each other (Figure 4A). For ADAMTS1, the upstream -3KB promoter region contained a total of 4 potential ETS binding sites, with two sites with in $114 \mathrm{bp}$ of each other (Figure 4A). A chromatin immunoprecipitation (ChIP) assay was performed by overexpressing ERG or vector control in PC3 cells, demonstrating direct binding of ERG to the promoter region for both CXCR4 and ADAMTSI (Figure 4A). Other regions of the CXCR4 and $A D A M T S 1$ promoter containing potential ERG binding sites were evaluated however none of these areas were different from controls in our ChIP assay. We further demonstrated in replicate ChIP experiments that ERG binds to the promoter region of CXCR4 (Figure 4B). To further evaluate the role of CXCR4 in promoting cell migration in PC3 cells overexpressing ERG, siRNA knock-down of CXCR4 and control GFP was used (Supplementary Figure 2C). Knockdown of CXCR4 demonstrated inhibition of cell migration (Figure 4C). Furthermore, we found in vivo that prostate specific ERG over-expression was associated with transcriptional up-regulation of $\mathrm{Cxcr} 4$ and to a lesser extent Adamts 1 compared to controls (Figure 4D and Supplementary Figure 2D). Additionally, in Pten loss prostate tumorigenic models that selected for Erg upregulation, Cxcr4 transcript levels were also upregulated (Figure 4E). While previous publications have not found a biunivocal correlation of ETS genetic rearrangements and CXCR4 expression levels in human prostate cancer specimens, ETS transcription factors may be only one of the pathways by which CXCR4 levels are regulated. ${ }^{6,7}$ Highlighting this is the fact that over $80 \%$ of primary prostate cancers display up-regulation of CXCR4 compared to normal prostate epithelium. ${ }^{24}$

In summary, ERG genetic rearrangements and loss of PTEN are frequent concomitant events in prostate cancer. We have demonstrated that Pten haploinsufficiency cooperates with $E R G$ aberrant expression to accelerate initiation and promote progression of prostatic adenocarcinoma. Pten haploinsufficiency has been demonstrated to promote cell proliferation and the development of prostatic intra-epithelial neoplasia, however, these lesions do not acquire the properties to progress to invasive disease. ${ }^{12}$ However, when Pten haploinsufficiency occurs concomitantly with $E R G$ aberrant expression, the role of $E R G$ on cell migration and invasion rapidly promotes progression of HGPIN to invasive cancer. However, given that we also observe acceleration of disease initiation in our Pten ${ }^{+/-}$PB$E R G$ mouse model, there are likely to be other cellular processes regulated by ERG, yet to be defined, which may cooperate with PTEN loss to promote the initiation of the PIN lesion, such as effects on cell survival, differentiation, and DNA repair mechanisms.

Our analysis demonstrates that two frequent critical events in human prostate cancer cooperate to promote tumor development and progression in the prostate. In turn this new knowledge will allow for more accurate patient stratification towards target therapies and prognostication. In fact, Yoshimoto et al. recently demonstrated that the occurrence of these two genetic events in patients with prostate cancer has a significant clinical impact. ${ }^{16}$ This study demonstrated that the co-occurrence of PTEN loss and ERG genetic rearrangement was a statistically independent predictor of biochemical failure after radical prostatectomy. ${ }^{16}$ Most importantly, the identification of ERG as a cooperative initiation event in prostate 
tumorigenesis suggests that ERG targeted therapies, when feasible, may be effective at preventing the transition between HGPIN and invasive cancer, while pharmacological manipulation of the PTEN/PI3K/AKT pathway may represent a powerful chemopreventive and chemotherapeutic tool in the future. Our findings also suggest that targeted therapies against these critical and frequent events should be tested combinatorially in the future and our current mouse model will provide an effective tool in studying pre-clinically such combinatorial modalities.

\section{Methods}

\section{Tumor Tissue Microarray}

Tumor tissue microarrays were generated for 40 prostate cancer specimens in triplicate and consecutive sections were obtained. FISH was carried out on tissue microarrays constructed from cases of prostatic carcinoma with matched high grade prostatic intraepithelial neoplasia. Approval was obtained from the institutional review board (IRB) and the Human Tissue Utilization Committee (HTUC) prior to study of human specimens. Break apart FISH probes consisted of 2 BAC clones each at 5'ERG ( RP11-55G21 and RP11-110N12), 3'ERG (RP11-315E22 and RP11-720N21) with approximately a $123.35 \mathrm{~kb}$ genomic gap between the two sets; and 2 BAC clones each at 5' TMPRSS2 (RP11-35C4 and RP-891L10) and 3' TMPRSS2 (RP11-825A8 and RP11-120C17), with an approximately $346.9 \mathrm{~kb}$ gap between the two sets. DNA was labeled by nick translation using SpectrumOrange-dUTP ( $3^{\prime}$ clones) or SpectrumGreen-dUTP (5' clones) (Vysis, Abbott Molecular Inc., Des Plaines, IL) Tissue hybridization, washing and fluorescence detection were performed as previously described (Leversha, 2001, Methods Mol Biol v.175) and according to standard procedures. Samples were analyzed using an automated imaging system with an Axioplan2 fluorescence microscope and scanning stage (Marzhauser) controlled by Metafer 4.0 and Isis 5.0 scanning and imaging software (MetaSystems, Altlussheim, Germany).

PTEN (PTEN Rabbit mAB 9559, Cell Signaling) immunohistochemical staining was performed by our molecular cytology core facility.

\section{Generation of plasmid vectors and transgenic construct}

Human $E R G$ cDNA sequence (transcript variant 1, REFSEQ NM_182918.2) was obtained from the NCBI database and primers (Supplementary Table 1) were designed to amplify the entire coding sequence. RNA obtained from 293HEK cells was utilized to amplify the human $E R G$ coding sequence, which was subsequently cloned into a TOPO vector (Invitrogen). Sequence analysis was performed to verify appropriate coding sequence and reading frame. This was then subcloned into a Flag-tag vector (CMV-Tg2B) using restriction enzyme digest with Pst1 and Xho1 in a standard fashion. We further subcloned our construct into the MSCV-PIG with the puromycin resistance gene (Xho1) plasmid and the PB-SV40 (Xho1) plasmid using Xho1 restriction digest in a standard fashion. The MSCV-AKT (constitutively active mutant) with the hygromycin resistance gene was obtained from Charles Sawyers. These constructs were amplified according to standard mini prep and maxi prep protocols. All constructs generated were screened by sequence analysis to verify appropriate coding sequence and reading frame.

\section{Establishment of probasin-ERG mouse colony, genotyping, phenotyping, and tissue analysis}

To determine the in vivo causal significance of ERG over-expression in prostate tumorigenesis, we generated a mouse model expressing ERG in a prostate specific manner. To accomplish this, the human $E R G$ cDNA sequence was cloned into a Flag-tag vector, subcloned into a probasin (ARR2PB) - SV40 expression vector, (Supplementary Figure 1A) and 
transgenic mice (B16 background) were established with assistance from our genetically engineered mouse core facility. Mice were subsequently analyzed for construct integration by Southern blot analysis (Supplementary Figure 1B). A total of 8 founders, positive for the $E R G$ transgene by Southern blot analysis were identified, and F1 offspring were established. To ensure similar passage of the transgene to future progeny, Southern blot analysis was performed on our F1 offspring, revealing similar patterns and intensity for 7 of the 8 founder lines (Supplementary Figure 1B). The founding line with dissimilar transgene passage was euthanized and excluded from the study. Additionally, a PCR genotyping assay was established and validated for analysis of future progeny. Briefly, primers (Supplementary Table 1) were designed to amplify the $3^{\prime}$ region of probasin and the $5^{\prime}$ region of $E R G$ and protocol was followed using HOT Star Taq Master Mix Kit (Qiagen) (Supplementary Figure 1C). Our PCR genotyping correlated with our Southern blot analysis in all cases.

Prostate specific expression of human $E R G$ was evaluated at the mRNA and protein levels by quantitative RT-PCR and flag immunohistochemistry, respectively. Overall, 5 of the 7 original founding lines demonstrated mRNA and 3 lines showed protein expression of human ERG in all lobes of the prostate with highest expression in the ventral prostate lobe, dorsal lateral lobe, and lowest levels of expression in the anterior lobe. (Supplementary Figure 1D, 1E). We did not observe any detectable transgene levels in the liver, spleen, lung, or tail of these mice. There was no difference in murine Erg expression among our founders and controls (Supplementary Figure 1D). Founding lines labeled A and B were subsequently expanded for phenotypic analysis and crossed into our Pten heterozygous mice to generate F2 offspring with the genotypes Pten ${ }^{+/+}$, Pten $^{+/-}$, Pten $^{+/+}$PB-ERG, and Pten ${ }^{+/-}$PB-ERG. ${ }^{12}$

All mouse work was performed in accordance with our IACUC protocol. Mice were euthanized using carbon-dioxide asphyxiation, and prostate tissues were procured for formalin fixation, paraffin embedding, and frozen storage for future molecular analyses. All tissue processing, paraffin embedding, section, and hematoxylin and eosin (H\&E) staining was performed by Histoserv Inc. Protein from the prostate specimens was extracted by homogenizing the tissue in RIPA buffer. RNA from the prostate specimens was extracted by homogenizing the tissue in TRIzol (Invitrogen). F2 offspring were generated for 2 independent transgenic lines (Lines A and B) and analyzed for prostate phenotype, with tissue procurement for evaluation as previously described. Results were similar between the two transgenic lines and all data is reported for transgenic line A.

\section{Murine immunohistochemistry protocols and antibodies}

All immunohistochemistry procedures were performed through our molecular cytology core facility with previous established protocols for Flag antibody (Santa Cruz), Ki67 antibody (Novocastra), PTEN antibody (Ab-2; NeoMarkers), and phospho serine-473 AKT antibody (Cell Signaling).

\section{Microarray analysis}

Microarray analysis was performed by our genomics core lab microarray facility. Briefly, MSCV-ERG and vector control were transiently expressed in 293 HEK cells for 2 independent experiments. RNA was harvested and subsequently quantified and labeled by our core department. Microarray analysis was performed on the U133A Affymetrix chip. Results were analyzed using GeneSpring software for 2 fold differences in gene expression across the 2 independent experiments. Results were validated with quantitative RT-PCR. Our complete microarray expression data set is uploaded on GEO-NCBI accession number GSE14595. 


\section{Quantitative RT-PCR protocol and primer sets}

Quantitative RT-PCR was performed using the SYBR Green PCR light cycler protocol (Qiagen). Subsequently, cDNA was generated from the RNA using the Super Script III First-Strand Synthesis system for RT-PCR (Invitrogen). See Supplementary Table 1 for primer sequences. Analysis was performed accordingly throughout our experiments and results were normalized to the house keeping genes ACTIN and Hprt for human and mouse specimens respectively.

\section{Western blotting protocol and antibodies}

Protein lysates were prepared using RIPA buffer and protease inhibitor cocktail (Roche). The following antibodies were used for western blotting: Flag antibody (Santa Cruz), Actin (AC-74; Sigma), ERG (H-95; Santa Cruz), phospho serine-473 AKT antibody (Cell Signaling), and phospho 240/244 S6 (Cell Signaling).

\section{In vitro analysis of cell phenotype}

Cell lines were established from primary Mef with our MSCV-ERG and MSCV puromycin selection vectors through initial infection and selection with puromycin. Stable clones were established from PC3 and 293 cells with our MSCV-ERG and MSCV puromycin selection vectors through initial transfection and selection with puromycin. BPH-1 cell lines were obtained from Simon Hayward and infected with MSCV-ERG, MSCV, MSCV-AKT (constitutively active $A K T-1$ ), and MSCV-AKT MSCV-ERG and selected with puromycin and hygromycin. These cells were then used for a variety of cellular assays and all assays were performed in triplicate. Protein and RNA were harvested using standard protocols for molecular analyses. For cell proliferation, $1 \times 10^{4}$ cells were plated in 12 well dishes, and cells were trypsinized and counted using a hemocytometer on days $2,4,6$, and 8 . Cell migration assay was performed by incubating $1 \times 10^{4}$ cells on a 8.0 um 24 well plate chamber insert (Falcon) with $0.9 \%$ FCS cell media above the insert and cell media containing 10\% FCS below the insert to establish a gradient. Cells were incubated for 24 hours and subsequently fixed with $4 \%$ paraformaldehyde and washed with PBS. Cells adherent to the top of the insert were scraped free with a cotton swab, washed with PBS and cells adherent to the bottom of the insert were stained with crystal violet blue. Positive staining cells were counted across 3 high-powered fields (100X).

\section{Chromatin immunoprecipitation (ChIP) protocol}

Our protocol was adapted from Strahl-Bolsinger et al as previously described. ${ }^{25}$ Briefly, PC 3 cells were transfected with $\mathrm{Tg} 2 \mathrm{~b}-E R G$ and harvested after 48 hours. The cells were fixed using $1 \%$ formaldehyde and subsequently washed with PBS. Cells were suspended in ChIP lysis buffer and sonicated at level 2 for 30 seconds. Immunoprecipitation was performed with the Flag antibody (Santa Cruz) or control IgG, and beads were washed with 4x ChIP lysis buffer, 2x ChIP wash buffer, and 2x Tris buffer, with pelleting of the beads. Immunoprecipitants were eluted in 150 ul of ChIP elution buffer, incubated, and DNA was eluted using the Qiagen PCR purification protocol. Primers (Supplementary Table 1) were designed to amplify regions of the CXCR4 and ADAMTS1 promoter spanning the putative ETS binding sites.

\section{CXCR4 knock-down}

Pooled siRNA against CXCR4 and GFP control was obtained from Dharmacon and knockdown evaluation was performed by qRT-PCR for $C X C R 4$. PC3 cells over-expressing ERG were transfected with $C X C R 4$ siRNA and control and a cell migration assay was performed as previously described. 


\section{Supplementary Material}

Refer to Web version on PubMed Central for supplementary material.

\section{Acknowledgments}

We are grateful to all members of the Pandolfi laboratory and Dr. Charles Sawyers and his group for stimulating intellectual discussions regarding our study project. We would like to thank Drs. P. Romanienko and W. Mark from our genetically engineered mouse core facility for their assistance in generating and genotyping our prostate specific ERG mouse model. We also would like to thank Simon Hayward for providing us with the BPH-1 cells used for our experiments, Ms. R. Lester at our animal facility for caring for our mice on a daily basis, and Ms. L. DiSantis for her editorial support. This work was funded through grants from the NIH (R01-CA82328, R01CA84292, P50-CA92629), and MSKCC Research in Therapeutics Program in Prostate Cancer award to B.S.C. and P.P.P.

\section{References}

1. Tomlins SA, et al. Recurrent fusion of TMPRSS2 and ETS transcription factor genes in prostate cancer. Science 2005;310:644-648. [PubMed: 16254181]

2. Tu JJ, et al. Gene fusions between TMPRSS2 and ETS family genes in prostate cancer: frequency and transcript variant analysis by RT-PCR and FISH on paraffin-embedded tissues. Mod. Pathol 2007;20:921-928. [PubMed: 17632455]

3. Perner S, et al. TMPRSS2-ERG fusion prostate cancer: an early molecular event associated with invasion. Am. J. Surg. Pathol 2007;31:882-888. [PubMed: 17527075]

4. Hermans KG, et al. Two unique novel prostate-specific and androgen-regulated fusion partners of ETV4 in prostate cancer. Cancer Res 2008;68:3094-3098. [PubMed: 18451133]

5. Helgeson BE, et al. Characterization of TMPRSS2:ETV4 and SLC45A3:ETV5 gene fusions in prostate cancer. Cancer Res 2008;68:73-80. [PubMed: 18172298]

6. Tomlins SA, et al. Role of the TMPRSS2-ERG gene fusion in prostate cancer. Neoplasia 2008;10:177-188. [PubMed: 18283340]

7. Tomlins SA, et al. Distinct classes of chromosomal rearrangements create oncogenic ETS gene fusions in prostate cancer. Nature 2007;448:595-601. [PubMed: 17671502]

8. Mosquera JM, et al. Characterization of TMPRSS2-ERG fusion in high-grade prostatic intraepithelial neoplasia and potential clinical implications. Clin Cancer Res 2008;14:3380-3385. [PubMed: 18519767]

9. Bettendorf O, et al. Chromosomal imbalances, loss of heterozygousity, and immunohistochemical expression of TP53, RB1, and PTEN in intraductal cancer, intraepithelial neoplasia, and invasive adenocarcinoma of the prostate. Genes Chromosomes Cancer 2008;47:565-572. [PubMed: 18383208]

10. Gray IC, et al. Mutation and expression analysis of the putative prostate tumour-suppressor gene PTEN. Br. J. Cancer 1998;78:1296-1300. [PubMed: 9823969]

11. Whang YE, et al. Inactivation of the tumor suppressor PTEN/MMAC1 in advanced human prostate cancer through loss of expression. Proc. Natl. Acad. Sci. U S A 1998;95:5246-5250. [PubMed: 9560261]

12. Di Cristofano A, Pesce B, Cordon-Cardo C, Pandolfi PP. Pten is essential for embryonic development and tumour suppression. Nat. Genet 1998;19:348-355. [PubMed: 9697695]

13. Trotman LC, et al. Pten dose dictates cancer progression in the prostate. PLoS. Biol 2003;1:385396.

14. Chen Z, et al. Crucial role of p53-dependent cellular senescence in suppression of Pten-deficient tumorigenesis. Nature 2005;4:725-730. [PubMed: 16079851]

15. King JC, et al. Cooperativity of TMPRSS2-ERG with PI3-kinase pathway activation in prostate oncogenesis. Nat. Genet. 2009 in press.

16. Yoshimoto M, et al. Absence of TMPRSS2:ERG fusions and PTEN losses in prostate cancer is associated with a favorable outcome. Mod Pathol. May 23;2008 (Epub ahead of print). 
17. Arya M, Ahmed H, Silhi N, Williamson M, Patel HR. Clinical importance and therapeutic implications of the pivotal CXCL12-CXCR4 (chemokine ligand-receptor) interaction in cancer cell migration. Tumour Biol 2007;28:123-131. [PubMed: 17510563]

18. Krampert M, et al. ADAMTS1 proteinase is up-regulated in wounded skin and regulates migration of fibroblasts and endothelial cells. J. Biol. Chem 2005;280:23844-23852. [PubMed: 15843381]

19. Salvucci O, et al. The role of CXCR4 receptor expression in breast cancer: a large tissue microarray study. Breast Cancer Res. Treat 2006;97:275-283. [PubMed: 16344916]

20. Ratajczak MZ, et al. The pleiotropic effects of the SDF-1-CXCR4 axis in organogenesis, regeneration and tumorigenesis. Leukemia 2006;20:1915-1924. [PubMed: 16900209]

21. Arya M, et al. The importance of the CXCL12-CXCR4 chemokine ligand-receptor interaction in prostate cancer metastasis. J. Exp. Ther. Oncol 2004;4:291-303. [PubMed: 15844659]

22. Hart CA, Brown M, Bagley S, Sharrard M, Clarke NW. Invasive characteristics of human prostatic epithelial cells: understanding the metastatic process. Br. J. Cancer 2004;92:503-512. [PubMed: 15668715]

23. Maroni P, Bendinelli P, Matteucci E, Desiderio MA. HGF induces CXCR4 and CXCL12-mediated tumor invasion through Ets1 and NF-kappaB. Carcinogenesis 2007;28:267-279. [PubMed: 16840440]

24. Xing Y, et al. Tumor cell-specific blockade of CXCR4/SDF-1 interactions in prostate cancer cells by hTERT promoter induced CXCR4 knockdown. Cancer Biol Ther 2008;7:1840-1849.

25. Strahl-Bolsinger S, Hecht A, Luo K, Grunstein M. SIR2 and SIR4 interactions differ in core and extended telomeric heterochromatin in yeast. Genes Dev 1997;11:83-93. [PubMed: 9000052] 
A
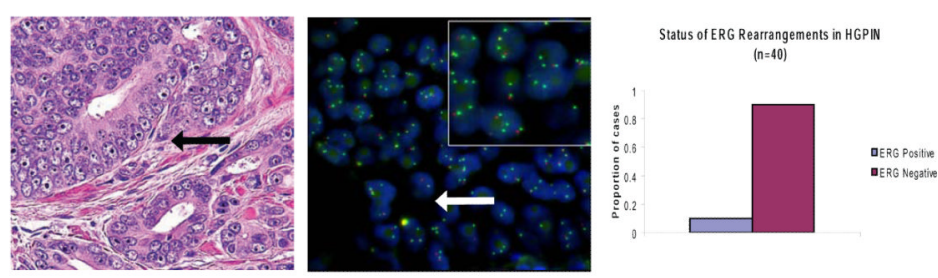

B

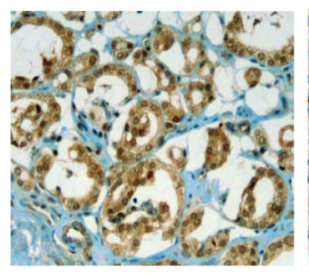

Normal PTEN Levels

Weak/Absent PTEN Levels

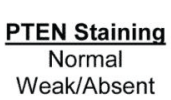

ERG FISH

Negative $(\mathbf{n}=\mathbf{2 5})$

12
13

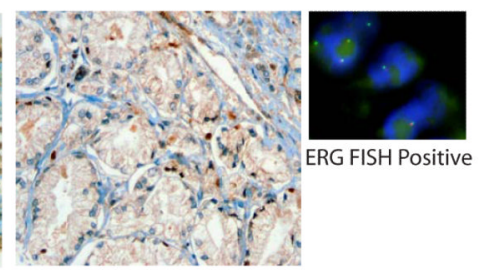

ERG FISH

Positive $(n=15)$

1

$p=0.007$

Figure 1. Genetic and molecular alterations ERG and PTEN are frequent and concomitant events in human prostate cancer

FISH break apart probes to 3' (red) and 5' (green) ends of both ERG and TMPRSS2 were used. Microscopy (200X), showing hematoxylin and eosin staining of HGPIN (arrowhead) and prostate carcinoma juxtaposed, high grade PIN (arrowhead) with deletion of the $3^{\prime}$ TMPRSS2 region, and prostatic adenocarcinoma (insert) with deletion of the 3'TMPRSS2 region, as seen by loss of the red signal in at least one of the red-green pairs (A). Bar graph shows the proportion of HGPIN cases by ERG status (A). Tumor tissue microarray demonstrating prostate cancer with normal PTEN staining and prostate cancer with weak/ absent PTEN staining (B). FISH staining from consecutive sections of the same specimen demonstrating the ERG genetic rearrangement is shown. The table reports the frequency of PTEN status by ERG rearrangement status (B). 
A

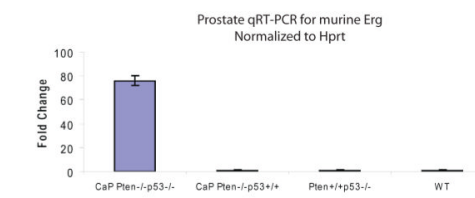

B

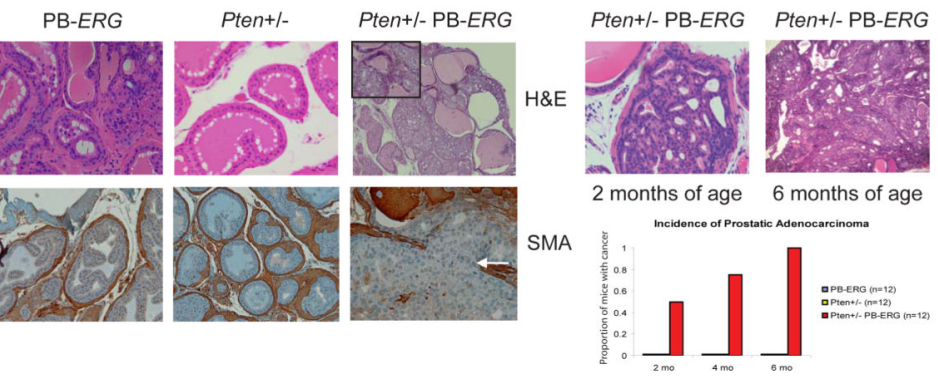

C

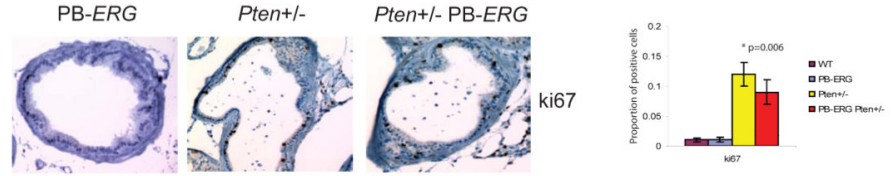

D

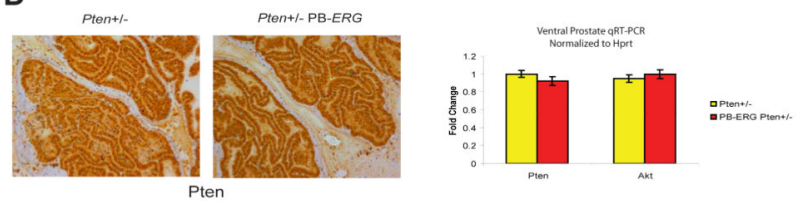

Figure 2. Prostate specific over-expression of ERG cooperates with Pten haploinsufficiency to promote prostate tumorigenesis

Locally advanced mouse $\left(\mathrm{Pten}^{-/-} \mathrm{p53^{-/- }}\right.$ ) prostate cancer demonstrated increased expression of murine Erg at the transcript level (normalized to Hprt) compared to controls (A). A total of 12 Pten $^{+/-}, 12$ ERG transgenic mice, and 12 Pten $^{+/-}$ERG mice were phenotypically characterized. High $(200 \mathrm{X})$ power histologic demonstration of prostatic adenocarcinoma in Pten $^{+/-}$ERG mice compared to controls in mice 6 months of age (B). Immunohistochemistry for smooth muscle actin is performed demonstrating invasion (B). Phenotypic characterization of $\mathrm{Pten}^{+--}$ERG mice at 2 and 6 months of age (200X). The incidence of prostatic adenocarcinoma is reported for transgenic mice at mice 2, 4, and 6 months of age (B). A bar graph is shown demonstrating the percentage of mice at each time point with the histologic finding of prostatic adenocarcinoma. Immunohistochemistry for Ki67 staining in pre-neoplastic prostate glands (mice 4 months of age) demonstrated no increase in proliferation for transgenic $E R G$ mice while Pten haploinsufficiency provides a proliferative advantage (C). A bar graph is shown demonstrating the percentage of ki67 positive staining cells per gland with mean and standard deviation reported for 3

representative prostate glands with p-value calculated based on Chi-square statistic (C). Immunohistochemistry of Pten revealed no differences in staining for pre-neoplastic prostate glands at 4 months of age and prostate in vivo transcript levels for Pten and AKT were similar for both genotypes (D). 
A

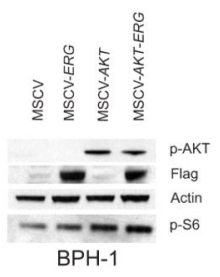

B

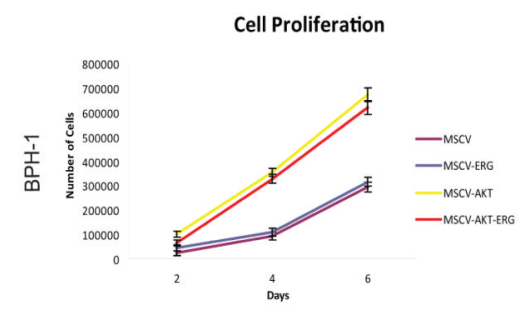

C

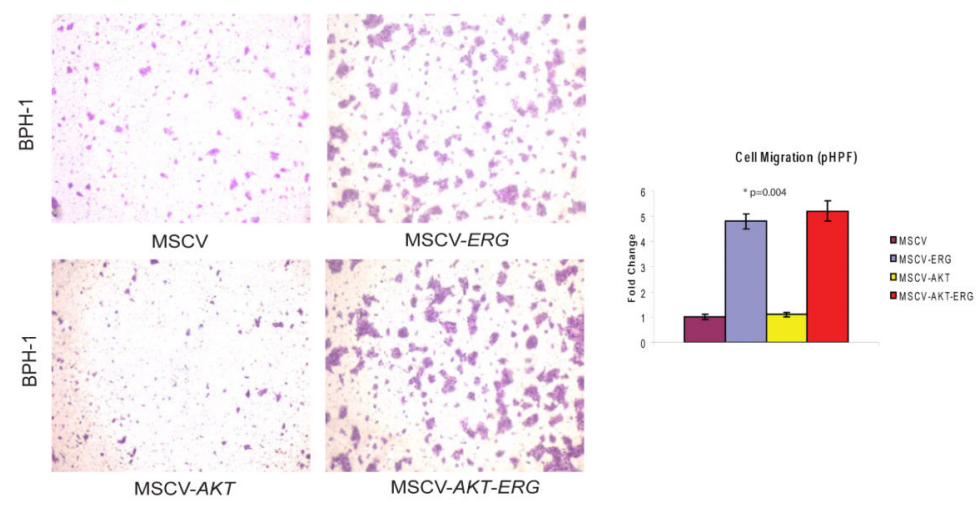

Figure 3. ERG regulates cell migration

Western blot analysis determined expression of Flag-ERG construct and activation of PAKT in stable BPH-1 cell lines infected with MSCV, MSCV-ERG, MSCV-AKT (AKT-1), and MSCV-AKT MSCV-ERG (A). While over-expression of a constitutively active AKT (AKT-1) promoted cell growth, no proliferative advantage was observed with ERG overexpression (B). A line graph is utilized to demonstrate cell proliferation with the mean and standard deviation represented from 3 experiments (B). A striking increase was observed in cell migration with over-expression of ERG compared to vector control in BPH-1 cells which was not augmented with over-expression of a constitutively active AKT (AKT-1) (C). Bar graphs demonstrate the fold change in the number of migrated cells per high powered field normalized to vector control, and the mean and standard deviation from 3 experiments were calculated with p-value calculated based on total number of cells migrating (C). 
A

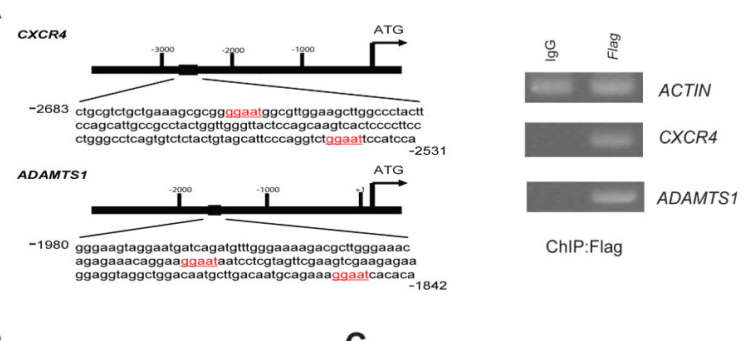

B

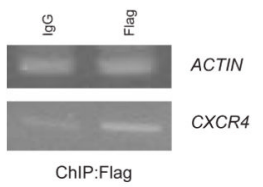

C

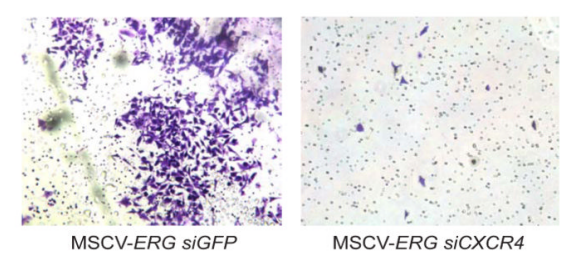

D

E
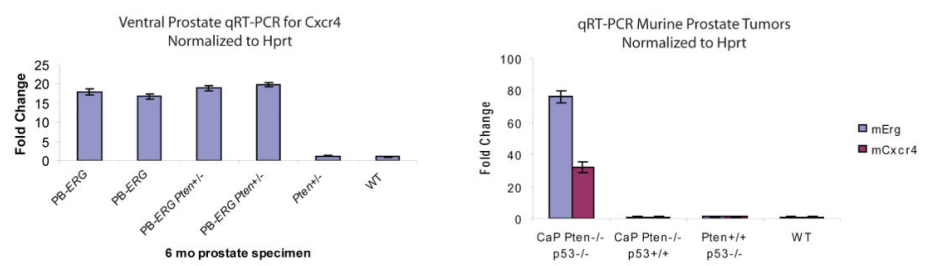

Figure 4. ERG directly regulates CXCR4 and ADAMTS1

Putative ETS binding sites were identified in the promoter regions of both human $C X C R 4$ and ADAMTS1 genes (A). ChIP assays were performed demonstrating direct ERG binding to the promoter regions for both $C X C R 4$ and ADAMTS1 (A). Repeat ChIP experiment for CXCR4 in PC3 cells over-expressing ERG (B). CXCR4 siRNA knock-down demonstrates a significant reduction in cell migration in PC3 cells over-expressing ERG (C). Further evaluation revealed that the mRNA expression of murine Cxcr4 was up-regulated in the prostate specimens of ERG transgenic mice (D) and Pten loss models of prostate tumorigenesis with up-regulation of ERG compared to controls (E). The bar graphs demonstrate the fold change in mRNA level following normalization to Hprt and our lowest value, and the mean and standard deviations from 3 experiments are shown. 


\section{Table 1}

Genes associated with tumorigenesis found to be up-regulated in 293 HEK cells by microarray expression analysis.

\begin{tabular}{cc}
\hline Gene & Fold Change \\
ATF5 & 3.004 \\
V-maf & 2.335 \\
ADAMTS1 & 2.102 \\
Jun B & 2.036 \\
CXCR4 & 2.029 \\
\hline
\end{tabular}

